\title{
Famílias com adolescente com síndrome de Down: apoio social e recursos familiares
}

\author{
Families with fidolescents with Down Syndrome: Social Support \\ and Family Resources \\ familias con adolescentes con síndrome de Down: apoyo social \\ y recursos familiares
}

\author{
Nara Liana Pereira-Silva, Larissa Dias Oliveira, Mayse Itagiba Rooke* \\ Universidade Federal de Juiz de Fora, Brasil
}

Doi: dx.doi.org/10.12804/apl33.02.2015.07

\section{Resumo}

O presente estudo tem os seguintes objetivos: (a) descrever a dinâmica e funcionamento de famílias com adolescentes com Síndrome de Down, bem como as reações e sentimentos dos genitores frente ao diagnóstico; (b) verificar a adequação dos recursos disponíveis às famílias, e (c) identificar a rede social de apoio percebida pelos genitores. Participaram do estudo quatro famílias compostas por pai, mãe e uma filha com síndrome de Down (SD), com idade entre 14 e 20 anos. Os resultados indicam que as mães são as principais responsáveis pelas tarefas da casa e cuidados com a filha com SD. Os escores alcançados na Escala de Apoio da Família e na Escala de Recursos da Família evidenciaram que essas famílias não têm apoios e recursos em níveis satisfatórios, ao mesmo tempo indicando a necessidade da implementação de programas de intervenção dirigidos ao grupo nessa fase do curso de vida.
Palavras chave: família; síndrome de Down; adolescentes; apoio social; recursos familiares.

\section{fbstract}

The present study has the following objectives: (a) describing the dynamics and functioning of families with adolescents with Down syndrome, as well as the reactions and feelings of parents facing the diagnosis; (b) verifying the adequacy of the resources available to families, and (c) identifying the social network of support perceived by parents. Four families participated in this study composed of father, mother and a daughter with Down syndrome (DS), aged between 14 and 20 years. The results indicate that mothers are the main responsible for housework and care of her daughter with SD. The scores achieved on the Family Support Scale and Family Resources Scale indicate that these families don't have support and resources at satisfactory levels,

* Nara Liana Pereira-Silva, Departamento de Psicologia, Universidade Federal de Juiz de Fora, Brasil; Larissa Dias de Oliveira, Departamento de Psicologia, Universidade Federal de Juiz de Fora; Mayse Itagiba Rooke, Departamento de Psicologia, Universidade Federal de Juiz de Fora.

A correspondência relacionada com este artigo deve ser direcionada a: Nara Liana Pereira Silva, Departamento de Psicologia, Instituto de Ciências Humanas, Universidade Federal de Juiz de Fora, Campus Universitário, Rua José Lourenço Kelmer. CEP: 36036-900 - Juiz de Fora, Minas Gerais, Brasil. Correio eletrônico: naraliana.silva@ufjf.edu.br

Para citar este artículo: Pereira-Silva, N. L., Oliveira, L. \& Rooke, M. (2015). Famílias com adolescente com síndrome de Down: apoio social e recursos familiares. Avances en Psicología Latinoamericana, 33(2), 269-283. doi: dx.doi.org/10.12804/ ap133.02.2015.07 
at the same time indicating the necessity of implementing intervention programs directed to the family at this stage of the life course.

Key words: family; Down syndrome; adolescents; social support; family resources.

\section{Resumen}

El presente estudio tiene los siguientes objetivos: (a) describir la dinámica y el funcionamiento de familias con adolescentes con síndrome de Down, así como las reacciones y sentimientos de los progenitores frente al diagnóstico; (b) verificar la adecuación de los recursos disponibles en las familias, e (c) identificar la red social de apoyo percibida por los progenitores. Participaron del estudio cuatro familias, compuestas por padre, madre y una hija con síndrome de Down (SD), con edades comprendidas entre los 14 y los 20 años. Los resultados indican que las madres son las principales responsables por las tareas de la casa y cuidados con la hija con SD. Los puntajes alcanzados en la Escala de Apoyo de la Familia y en la Escala de Recursos de la Familia indican que esas familias no tienen apoyos y recursos en niveles satisfactorios; al mismo tiempo, indica la necesidad de la implementación de programas de intervención dirigidos al grupo en esa fase del curso de la vida.

Palabras clave: familia; síndrome de Down; adolescentes; apoyo social; recursos familiares.

O nascimento de uma criança com síndrome de Down (SD) exerce um forte impacto no sistema familiar, podendo afetar o grupo em diferentes dimensões (Bailey, 2007; Head \& Abbeduto, 2007; Henn, Piccinini \& Garcias, 2008), uma vez que este acontecimento não é um evento normativo. Nesse sentido, as famílias podem experienciar estresse, dificuldades de adaptação, além de restrições diversas, tais como as sociais (Cramm \& Nieboer, 2011; Dabrowska \& Pisula, 2010; Eisenhower \& Blacher, 2006; Falkenbach, Drexsler \& Werler, 2008; Goitien \& Cia, 2011; Hastings, Daley \& Burns, 2006; Henn \& Piccinini, 2010; Henn et al., 2008).
Em uma revisão de nove artigos acerca de famílias de crianças com deficiência, Bailey (2007) concluiu que a experiência de ter um filho com deficiência, frequentemente, causa impactos significativos na família, apesar das respostas a esse evento variarem entre os membros familiares. Dentre as variáveis que podem minimizar os efeitos desse impacto, pode-se mencionar a rede de apoio/suporte social. Ressalta-se que a natureza e a qualidade do suporte social podem estar relacionadas a uma adaptação positiva. Assim, além da dinâmica de funcionamento interno da família ser considerada um aspecto fundamental na adaptação à criança com deficiência intelectual (DI), a família está em interação mútua com sistemas externos, tais como a comunidade, a vizinhança, a rede social de apoio, dentre outros. Pesquisar essas influências é extremamente relevante para aprofundar na compreensão de fatores que envolvem o desenvolvimento humano (Bronfenbrenner, 2011; Dessen \& Braz, 2000) e, em especial, o das pessoas com deficiência (Hodapp, Burack \& Zigler, 1998).

Apesar do momento do nascimento de um filho ser um período de transição muito significativo no curso de vida familiar (por exemplo, Kreppner, 2000; Sá \& Rabinovich, 2006), deve-se evidenciar que, para as famílias com filhos SD, esse momento pode ser vivenciando com mais intensidade. Entretanto, destaca-se que todas as fases durante o curso de desenvolvimento da pessoa com SD e de sua família se tornam preponderantes, bem como suas interações com outros sistemas sociais, como tem sido destacado pelo modelo bioecológico de Bronfenbrenner (Bronfenbrenner \& Morris, 1998). A adoção desse modelo permite afirmar que a pessoa com SD recebe influências das diversas variáveis que compõem os níveis dos sistemas desde o microssistema até o macrossistema. Mas, a pessoa não somente recebe influências, ela também influencia o ambiente, em especial, o microssistema e suas alianças, nesse caso, os mesossistemas.

Não há como negar que a literatura, na área de família e DI, tem sido mais extensiva no tocante 
às famílias com filhos pequenos, não sendo muito abrangente quando a família tem filhos adolescentes (Barbosa, Pettengill, Farias \& Lemes, 2009; Cunningham, 1996; Hall, Bobrow \& Marteau, 1997; Most, Fidler, Laforce-Booth \& Kelly, 2006; Padeliadu, 1998; Stores, Stores, Fellows \& Buckley, 1998). É importante destacar que, no contexto brasileiro, a escassez de literatura com enfoque para famílias com filhos na adolescência (Pereira-Silva \& Oliveira, 2011) ainda é mais evidente. Por exemplo, no estudo de Pereira-Silva e Oliveira (2011) foram encontrados um total de 192 publicações relacionadas ao tema família e DI, sendo 171 estrangeiras e 21 brasileiras. Além disso, apenas 22 publicações estrangeiras abordavam a fase da adolescência, sendo $91 \%$ artigos de pesquisa e 9\% livros/capítulos de livros. Quanto às publicações brasileiras, somente 4 trabalhos correspondiam à adolescência, sendo todos artigos de pesquisa.

Sobre o período da adolescência, Bononi, Oliveira, Renattini, Sant'Anna e Coates (2009) afirmam que este é um período de desafios e confrontos para qualquer jovem, bem como para adolescentes com SD. Para estes autores, estes adolescentes vivenciam diferentes graus de isolamento social, o que limita as oportunidades de interação e de envolvimento afetivo. Além disso, as alterações na aparência física constituem fatores de risco para os problemas na adaptação social dos jovens com SD. No que se refere aos déficits sociais destes adolescentes, há relatos de que esses jovens experimentam problemas em estabelecer e manter relações com amigos na escola e com figuras de autoridade (Angélico, 2004; Soresi \& Nota, 2000). Tal fato diminui a qualidade e a quantidade de experiências sociais, podendo causar efeitos negativos nas habilidades de adaptação à vida adulta $\mathrm{e}$ na interação social.

Entretanto, há estudos que mostram dados positivos sobre adolescentes com $\mathrm{SD}$, não sendo esse período sinônimo apenas de fatores negativos. Por exemplo, Cunningham (2008) afirma que a maioria dos pais e mães de seu estudo relata que adolescentes e adultos jovens com SD são mais fáceis de lidar, mas também são menos ativos. Esse fato implica na diminuição da emissão de alguns comportamentos inadequados, tais como ser briguento, ser opositor e exigir atenção. Já no estudo de Bonomo, Garcia e Rossetti (2009), com 10 adolescentes com SD (entre 13 e 19 anos) e suas respectivas mães, verificou-se que os adolescentes mantêm um bom relacionamento com as pessoas. Do total de 10 mães, nove delas citaram características positivas para o bom relacionamento, tais como ser carinhoso, amoroso, alegre, doce, extrovertido, comunicativo, disposto e cativante.

Aliada a essas características, ressalta-se o fato de que a geração atual de adolescentes com SD teve oportunidade de frequentar centros de estimulação precoce, o que possibilitou um nível de desenvolvimento suficiente para a autonomia doméstica, apesar da maioria não ter condições de vida completamente independente (Casarin, 2003). Nesse sentido, Teixeira e Kubo (2008) destacam que o aumento de adolescentes com SD na escola tem propiciado maior condição de interação entre alunos com e sem DI, além de possibilitar o estreitamento dos vínculos afetivos entre esses alunos, redução de estereótipos em relação à síndrome, aquisição de respeito e valorização das diferenças, bem como a solidariedade entre os colegas.

Deve-se reconhecer que a adolescência representa um momento crítico que envolve toda a família, necessitando, portanto, considerar o adolescente, seus pais e todos os membros, tais como os irmãos e os avós (Scabini \& Ranieri, 2011). As famílias de adolescentes com SD, assim como aquelas de adolescentes com desenvolvimento típico (DT) também passam por mudanças. No entanto, o estudo de Bastos e Deslandes (2009) mostra que os genitores de adolescentes com SD têm dúvidas quanto a essa fase de desenvolvimento, uma vez que eles reconhecem apenas algumas características dos filhos associadas à adolescência, por exemplo, as transformações no corpo, mas não os consideram como adolescentes devido à pouca 
autonomia. Além disso, os genitores apresentaram dúvidas sobre como lidar com essa etapa do curso de vida e como contribuir para a independência nas atividades de vida diária do filho adolescente. Casarin (2003) acrescenta ainda a dificuldade da família em abordar alguns temas, por exemplo, a sexualidade, bem como lidar com a manifestação de tal aspecto do desenvolvimento, requerendo atenção especial por parte de profissionais e, também da rede social de apoio da família.

No tocante à rede social de apoio, esta constitui um importante sistema que pode auxiliar as famílias com pessoas com DI ou com SD ao longo do curso de vida, especialmente em seu processo de adaptação à criança com deficiência (Flynt, Wood, \& Scott, 1992). Para Colnago (2000), é a partir do apoio recebido que a família pode restabelecer suas condições emocionais e reunir forças para cuidar, educar e prestar os atendimentos necessários ao desenvolvimento da pessoa com deficiência.

Nesse sentido, Cunningham (1996) realizou um estudo longitudinal com 134 famílias de pessoas com SD no Reino Unido. Os resultados indicaram que, no período da adolescência, as mães diminuem o nível de satisfação com a vida devido ao declínio da satisfação com o suporte social. Ao longo do tempo, as mães passaram a perceber que seus filhos com SD viviam mais restrições do que os jovens sem deficiência.

No estudo de Barbosa et al. (2009) com seis mães de crianças com DI a família ampliada e os amigos apareceram como importantes fontes de suporte, fornecendo informações sobre o que acontece com o filho e seus cuidados; apoiando nos recursos materiais, estruturais e financeiros e, também, eram suportivos no tocante à avaliação, isto é, nas afirmações positivas recebidas pela equipe especializada. Esses resultados são similares aos de outros estudos envolvendo famílias de crianças com DT, por ocasião do nascimento de filhos (Dessen \& Braz, 2000; Oliveira \& Dessen, 2012), em que os familiares são considerados como a principal fonte de apoio.
Bastos e Deslandes (2008) destacam, ao analisar narrativas de mães de adolescentes com DI, a importância do apoio social enquanto fator que potencializa as condições da família, especialmente do ponto de vista afetivo e econômico. A análise das narrativas demonstrou que o momento do diagnóstico do filho teve um impacto importante para as famílias, envolvendo desde a falta de compreensão até a redefinição da identidade do filho, bem como a busca de estratégias de adaptação ao novo membro e da procura por apoio na rede pública de atendimento. Essas dificuldades refletem a limitação da rede de apoio institucional e de profissionais presentes na realidade da maioria das famílias participantes, refletindo o panorama brasileiro. As dificuldades encontradas por mães em relação ao suporte social também foram destacadas por Wodehouse e McGill (2009). Treze mães de pessoas com DI e problemas de comportamento relataram encontrar dificuldades de acesso aos serviços de atendimento ao filho, obstáculos na obtenção de informações relevantes e distanciamento na relação com os profissionais. Wodehouse e McGill (2009) destacam a necessidade de essas famílias ter maior acesso a serviços de prevenção, bem como de serem mais difundidas as técnicas de administração de comportamento; além da importância de haver uma melhora na relação entre famílias e profissionais.

Segundo Douma, Dekker e Koot (2006) a maioria dos genitores necessita de algum tipo de apoio social, principalmente, aqueles cujos filhos apresentam problemas emocionais e comportamentais associados à DI. Os resultados da investigação de Douma et al. (2006) mostram que pais e mães de jovens com DI tendem a vivenciar altos níveis de estresse parental, necessitando, assim, do suporte de uma rede social para melhor lidar no enfrentamento da situação. É interessante destacar que, segundo Douma et al., aqueles genitores que demonstraram maior necessidade de suporte recebiam apoio com maior frequência do que aqueles que demonstraram menos necessidade de suporte. 
No estudo de Matsukura, Marturano, Oishi e Borasche (2007) acerca do suporte social e o estresse em mães de crianças com necessidades especiais, os resultados apontam para o fato de essas famílias possuírem um menor número de pessoas em sua rede social. $\mathrm{Na}$ análise dos resultados foi encontrada uma relação negativa entre estresse e suporte social, isto é, quanto maior o nível de estresse, menor o número de pessoas da rede social dessas mães. Esse dado aponta para a importância da rede social de apoio como recurso no enfrentamento da adaptação e no lidar dessas famílias com a pessoa que apresenta algum tipo de necessidade especial, tal qual a SD ou a DI.

Vale salientar que não somente as famílias com pessoas com deficiência, com SD ou necessidade especial se beneficiam do apoio propiciado pela rede social, mas também outros tipos de configurações familiares ou grupos sociais, tais como as famílias com crianças com problemas de saúde (por exemplo, Araújo, Reichert, Oliveira \& Collet, 2011; Moraes \& Cabral, 2012), aquelas no período de gravidez ou nascimento de filhos (por exemplo, Dessen \& Braz, 2000; Jussani, Serafim \& Marcon, 2007; Oliveira \& Dessen, 2012), famílias com filhos pequenos (por exemplo, Dezoti, Alexandre, Tallmann, Maftum \& Mazza, 2013), os adolescentes (por exemplo, Diniz, Garcia-Dias, Neiva-Silva, Nieto \& Koller, 2012; Siqueira, Betts \& Dell'Aglio, 2006), os cuidadores (Amendola, Oliveira, \& Alvarenga, 2011). Desta forma, não se pode negar a importância dos fatores internos e externos que envolvem a dinâmica e funcionamento familiar e suas implicações para o desenvolvimento do grupo, bem como de cada um de seus membros, sendo a rede social de apoio um desses fatores.

Dada a escassez de literatura acerca de adolescentes com SD e suas famílias (Pereira-Silva \& Oliveira, 2011), o presente estudo tem os seguintes objetivos: (a) descrever a dinâmica e funcionamento de famílias com adolescente com SD, bem como as reações e sentimentos frente ao diagnóstico; (b) verificar a adequação dos recursos disponíveis às famílias; e (c) identificar a rede social de apoio percebida pelos genitores.

\section{Método}

\section{Participantes}

Participaram do estudo quatro famílias brasileiras compostas de pai, mãe e uma filha com SD, procedentes de duas cidades do interior de Minas Gerais, Juiz de Fora $(n=2)$ e Caxambu $(n=2)$. As famílias são identificadas com a letra $F$ seguida por um número, F1, F2 e assim por diante. As adolescentes são identificadas com a letra A seguida pelo número correspondente ao de sua família, assim A1 é a adolescente de F1, por exemplo. As participantes com SD tinham as seguintes idades: 14 $\operatorname{anos}(\mathrm{A} 1), 20$ anos (A2 e A3) e 18 anos (A4), tendo uma média de 18 anos. Com relação à escolaridade, duas adolescentes não frequentaram o ensino regular (A3 e A4), uma completou o ensino fundamental (A2) e a outra tinha o ensino fundamental incompleto (A1). Em relação aos atendimentos e atividades frequentados pelas adolescentes, destacaram-se os seguintes: fonoaudiologia (A1, A2), psicologia e psicopedagogia (A1), terapia ocupacional (A2) e atividades do centro de atendimento especializado (A2, A3 e A4).

Em relação às famílias, as mães tinham, em média, 59 anos e os pais 68,5 anos. Quanto à escolaridade e ocupação dos genitores, bem como a renda familiar, a Tabela 1 apresenta tais informações.

\section{Instrumentos}

Foram utilizados os seguintes instrumentos: Questionário de Caracterização do Sistema Familiar (Dessen, 2009), o qual investigou dados demográficos das famílias, caracterizando o sistema familiar. Escala de Recursos da Família, a qual foi traduzida por Nunes (2006) do original de Leet e Dunst (1994), é constituída de 29 itens, os quais medem a adequação de recursos que têm sido iden- 
Tabela 1

Caracterização das famílias, segundo a idade, ocupação e escolaridade dos genitores e renda familiar

\begin{tabular}{|c|c|c|c|c|c|c|c|}
\hline \multirow{2}{*}{ Família } & \multicolumn{3}{|c|}{ Mãe } & \multicolumn{3}{|c|}{ Pai } & \multirow{2}{*}{$\begin{array}{l}\text { Renda } \\
\text { familiar }\end{array}$} \\
\hline & Idade & Escolaridade & Ocupação & Idade & Escolaridade & Ocupação & \\
\hline $\mathrm{F} 1$ & 49 & Ensino médio & Não trabalha fora & 56 & Ensino Médio & Serviços técnicos & 3.20 \\
\hline F2 & 67 & Graduação & Aposentada & 73 & Graduação & Aposentado & 16.00 \\
\hline F3 & 61 & Ensino fundamental & Aposentada & 78 & Sem escolaridade & Aposentado & 1.93 \\
\hline F4 & 59 & Ensino fundamental & Não trabalha fora & 67 & Ensino fundamental completo & Aposentado & 1.93 \\
\hline
\end{tabular}

Nota. A idade está exposta em anos e a renda familiar em salários mínimos brasileiros à época da coleta de dados.

tificados como principais componentes do suporte intra e extrafamiliar, devendo ser indicada de acordo com uma escala do tipo Likert variando de 1 a 5 pontos (nunca adequado, raramente adequado, algumas vezes adequado, geralmente adequado, sempre adequado), além da resposta não se aplica (NA). Quanto maior é a pontuação, mais adequados são os recursos disponíveis à família. Escala de Apoio da Família é um instrumento traduzido por Nunes (2006) do original de Dunst, Jenkins e Trivette (1994), sendo composta por 18 itens para avaliar se os recursos potenciais de apoio têm sido suportivos às famílias, sendo indicada de acordo com uma escala do tipo Likert variando de 1 a 5 pontos (nunca colabora, algumas vezes colabora, geralmente colabora, colabora muito, colabora extremamente). Quando alguma das fontes de apoio indicadas não estava disponível para a família naquele período, a resposta foi não disponível (ND).

Além destes instrumentos, foi construído, pelas pesquisadoras, um checklist acerca das atividades de vida diária (AVD) realizadas pelas adolescentes. Trata-se de uma lista com 25 itens para assinalar, com o objetivo de identificar as atividades que as adolescentes desempenham de forma Independente, Com ajuda ou Totalmente dependente. Os itens abrangem aspectos dos cuidados pessoais (por exemplo: usar o banheiro, lavar o rosto, tomar banho, escovar dentes), da alimentação (por exemplo: prepara refeições, serve alimentos), das tarefas rotineiras diversas (por exemplo: arrumar sua cama, guardar seus objetos, fazer pequenas compras, utiliza dinheiro, utiliza telefone, toma remédio) e do transporte/locomoção (por exemplo: vai a lugares distantes, vai da casa até lugares rotineiros, anda pela vizinhança, atravessa a rua, utiliza transporte público). Apenas a mãe respondeu a esse instrumento. $O$ roteiro de entrevista com os genitores continha questões sobre o diagnóstico da filha e seus sentimentos atuais em relação à mesma.

\section{Procedimentos}

Todos os procedimentos éticos necessários à execução do estudo foram respeitados: aprovação pelo comitê de ética em pesquisa com seres humanos da Universidade Federal de Juiz de Fora sob o número 119/2011; apresentação do projeto de pesquisa aos dirigentes das instituições de educação especial e assinatura do Termo de Consentimento Livre e Esclarecido, por ambos os genitores, para a realização de pesquisa.

Inicialmente, as pesquisadoras entraram em contato com quatro instituições públicas e privadas de atendimento às pessoas com deficiência de Juiz de Fora-MG para a seleção dos participantes com SD e suas famílias. Das instituições visitadas, somente em duas havia famílias que atendiam aos critérios da pesquisa, isto é, pais e mães casados ou morando juntos, tendo, pelo menos, um filho biológico com SD, com idade entre 13 e 20 anos. Foram contatadas cinco famílias, mas apenas duas concordaram em participar do estudo. Diante da 
escassez de participantes, uma instituição de atendimento especializado, na cidade de Caxambu-MG, foi contatada e duas famílias aceitaram participar, totalizando, portanto, quatro famílias com uma filha com SD.

A coleta de dados ocorreu na residência dos participantes e consistiu em duas visitas. Na primeira, ambos os genitores, separadamente, responderam à Escala de Recursos da Família e à Escala de Apoio da Família, com duração aproximada de uma hora e 30 minutos. Na primeira escala, o genitor era solicitado a indicar o quão cada um dos recursos estava adequado para atender às necessidades da família, desde o início até o fim de um mês. Na segunda, o genitor foi solicitado a indicar o quão colaborador cada indivíduo ou grupo estava sendo para ele em termos de educação e cuidado com a filha nos últimos 6 meses. Na segunda visita, a mãe respondeu ao Questionário de Caracterização do Sistema Familiar, ao Checklist de Atividades de Vida Diária e, ambos os genitores e a filha adolescente responderam ao roteiro de entrevista semiestruturada.

A análise dos resultados seguiu as especificidades de cada instrumento utilizado. Os dados coletados por meio de escalas, questionário e checklist foram tabulados, permitindo a elaboração de análises por meio de estatística descritiva. As entrevistas foram gravadas em áudio, com duração média de uma hora e transcritas na íntegra. Posteriormente as entrevistas foram categorizadas utilizando-se análise de conteúdo (Bardin, 2011) seguindo os passos destacados: identificação dos temas nos relatos, construção de categorias seguindo os critérios de exclusão mútua, homogeneidade e pertinência, e, finalmente, a análise de categorias feita por um juiz. As categorias foram definidas operacionalmente (ver Oliveira, 2013), tendo sido construído um dicionário de definição e realizada a análise por um pesquisador com experiência em análise de conteúdo e conhecimentos na área de DI e família.

\section{Resultados}

Inicialmente, são descritas as características das famílias, focalizando sua dinâmica e funcionamento, bem como as reações e sentimentos dos genitores no momento do diagnóstico de SD da filha. As competências e habilidades em atividades de vida diária, identificadas pelas mães das adolescentes com SD são descritas em seguida. Posteriormente, os resultados acerca da satisfação com o apoio social recebido e com os recursos familiares disponíveis são apresentados.

\section{Caracterizando a dinâmica e funcionamento das famílias}

Verifica-se que em três famílias as mães eram responsáveis pelas atividades domésticas de limpar a casa e lavar/passar roupas. Em apenas uma família (F2) havia empregada doméstica e a mãe era a responsável por orientá-la. A atividade de cozinhar tinha como responsável principal a mãe em três famílias (F2, F3 e F4) e o pai em uma delas (F1). A tarefa de comprar comida era compartilhada por ambos os genitores somente em uma família (F2), nas demais ou era responsabilidade da mãe (F3) ou do pai (F1 e F4).

Em relação aos eventos sociais, identifica-se uma variedade nos relatos. As atividades religiosas, tais como missas/cultos, não são realizadas com frequência pelas famílias, por exemplo, F1 e F3 frequentam uma vez por semana, F2 e F4 menos de uma vez por mês. Já as festas religiosas são frequentadas com mais assiduidade pelos membros da família F3. A família F4 nunca frequenta esses eventos, sendo que F1 e F2 frequentam menos de uma vez por mês. Em relação às visitas familiares e aos amigos, F1, F2 e F3 as realizam menos de uma vez por mês, sendo que F4 nunca as realiza. Os diversos tipos de comemorações são mais frequentes para F1, F2 e F3, bem como as festas típicas, sendo que F4 nunca as frequenta. Os passeios ao 
cinema e teatro não são frequentes, por exemplo, F1 e F4 nunca vão a estes ambientes, mas F2 costuma frequentar menos de uma vez ao mês e F3 de uma a três vezes ao mês. Os centros culturais não são visitados por F3 e F4, sendo que F1 visita raramente e F2 de uma a três vezes ao mês. No geral, F4 é a família que relata menor frequência aos eventos sociais, participando apenas de missas/ cultos menos de uma vez por mês.

Quanto às tarefas relacionadas às filhas, todas as mães eram as principais responsáveis por: supervisionar o banho e a alimentação, bem como levar a filha para a escola. Essa última atividade, em duas famílias, era compartilhada com o pai (F2 e F3). Colocar a filha para dormir era realizado principalmente pela mãe (F2, F3 e F4) e somente por um pai (F1). No que tange à atividade de contar histórias, a mãe (F2), o pai (F4) e a tia (F3) a realizavam com frequência. Em F1 tal atividade não era realizada. Verifica-se que as mães eram as responsáveis pela maioria das atividades desenvolvidas com as filhas.

\section{Momento do diagnóstico: reações e sentimentos}

Todas as mães afirmaram que tiveram a confirmação do diagnóstico de SD da filha logo após o seu nascimento. As reações diante da confirmação do diagnóstico foram: Choro (mãe-F2), Ausência de Reação (pai - F1 e F4; mãe - F1) e Aceitação (pai-F2; mãe-F3 e F4). Apenas um pai (F3) não relatou explicitamente sua reação.

A respeito dos sentimentos vivenciados pelos genitores, a maioria relatou Tristeza $(n=6$; pai - F1, F3, F4; mãe - F1, F2, F3), seguido por Nervosismo ( $n=1$; mãe - F4) e Felicidade $(n=1$; pai-F2). Quanto aos sentimentos atuais dos genitores em relação à filha com $\mathrm{SD}$, foram relatados os seguintes: Insegurança (mãe - F3, F2), Carinho (mãe - F4; pai - F4) e Felicidade (pai - F2, F1). Dois genitores (mãe $-\mathrm{F} 1$; pai $-\mathrm{F} 3$ ) não relataram o que sentem atualmente. Diante da confirmação do diagnóstico, todas as mães relataram ter procurado ajuda profissional, sendo que em F1, F3 e F4 a mãe tomou a iniciativa de procurar tal ajuda sozinha e em F2, a iniciativa foi do casal. As famílias possuem pouco conhecimento sobre a SD, isto é, três mães (F1, F3 e F4) expressaram ter 'Conhecimento genérico' acerca da SD e apenas uma mãe (F2) relatou ter 'Conhecimento científico'.

\section{Competências e habilidades das adolescentes em atividades de vida diária}

No que se refere às habilidades das adolescentes, houve uma variedade nos resultados relatados pelas mães. As atividades que todas realizavam de maneira independente foram as seguintes: retirar a roupa, lavar as mãos e lavar o rosto. Os resultados indicaram que, das 25 atividades propostas, as participantes A1 e A4 realizavam oito delas de forma independente, enquanto $\mathrm{A} 2$ realizava sete $\mathrm{e}$ A3 realizava 16 atividades independentemente. As atividades realizadas Com ajuda foram mais frequentes para as adolescentes A1 e A2, respectivamente 13 e 11 atividades eram realizadas Com ajuda. Nas atividades realizadas de forma Totalmente dependente, verifica-se que todas as adolescentes realizavam as seguintes: preparar refeições, ir a lugares distantes e tomar remédios. As adolescentes A1 e A4 realizavam somente quatro atividades de forma totalmente dependente, enquanto A2 e A3 realizavam oito e 10 atividades, respectivamente.

\section{O apoio social e os recursos familiares}

Considerando a quantidade de participantes e o fato das escalas não possuírem padronização para a população brasileira, esses resultados devem ser analisados com cautela. A tabela 2 apresenta os escores referentes ao nível de colaboração da rede de apoio percebido pelos genitores, bem como à adequação dos recursos familiares disponíveis. Verifica-se que a família (F2) com níveis mais adequados de recursos possui menos colaboração de redes de apoio social. As famílias com níveis menos 
adequados de recursos (F1 e F4) são aquelas com maiores níveis de colaboração da rede de apoio.

Tabela 2

Escores referentes à colaboração recebida pela rede de apoio social e nível de adequação dos recursos familiares

\begin{tabular}{ccccccccc}
\hline & \multicolumn{3}{c}{ Apoio recebido } & \multicolumn{4}{c}{ Recursos familiares } \\
\cline { 2 - 9 } Genitor & F1 & F2 & F3 & F4 & F1 & F2 & F3 & F4 \\
\hline Mãe & 38 & 13 & 22 & 34 & 72 & 137 & 97 & 84 \\
Pai & 33 & 28 & 30 & 43 & 108 & 126 & 122 & 102 \\
\hline
\end{tabular}

Por meio da análise de cada família é possível identificar os componentes indicados por cada genitor como colaboradores. Para a família F1 os apoios recebidos da própria família e dos amigos não estão disponíveis, enquanto o apoio de profissionais é considerado mais colaborativo, segundo o pai. Para a mãe, nessa família, o apoio de profissionais também é considerado mais colaborativo, já os familiares do marido nunca colaboram. Já na família F2, ambos os genitores consideram o cônjuge e os filhos mais colaborativos. Entretanto, as seguintes formas de suporte foram consideradas não disponíveis: colegas de trabalho, grupos de pais, grupos sociais ou clubes, membros/ministros da igreja, agências de profissionais. Nas famílias F3 e F4, os genitores consideram os filhos os mais colaborativos. Membros/ministros da igreja e parentes mais próximos do marido ou companheiro foram apontados como menos colaborativos, na percepção da mãe em F3. Para o pai em F3, a rede de apoio composta pelos amigos, colegas de trabalho, grupos de pais, grupos sociais ou clubes, membros/ministros da igreja, médicos da família ou dos filhos foram considerados como menos colaborativos. Para ambos os genitores em F4 os amigos e outros pais foram considerados os menos colaborativos. Quanto ao apoio recebido para colaborar/auxiliar nos cuidados e na educação das filhas, verifica-se que F4 é a família que percebe o apoio como mais colaborador, enquanto F2 o percebe como menos colaborador. $\mathrm{O}$ escore referente ao pai em F4 e à mãe em F1 foram os mais elevados nessa escala, sendo o mais baixo o da mãe em F2.

A análise mais específica dos níveis de adequação dos recursos disponíveis às famílias permite afirmar algumas particularidades relativas a cada família, de acordo com cada genitor. Em F1, ambos os genitores consideraram adequados os recursos disponíveis para a moradia e alimentação. Entretanto, o pai não considera adequada apenas a assistência pública e as férias/viagem de férias e a mãe os recursos financeiros e aqueles relativos ao tempo gasto com a família e consigo mesma. Quanto à família F2, comparando-a com as demais, é a família mais satisfeita com os recursos. Mãe e pai consideram a maioria dos recursos sempre adequados para atender às suas necessidades. Nenhum dos recursos foi considerado 'nunca adequado' ou 'raramente adequado', por nenhum dos genitores. A família F3 considera os recursos 'sempre adequados' ou 'geralmente adequados'. Os recursos considerados menos adequados pela mãe foram apenas dinheiro para ser guardado e férias/viagem de férias. Já o pai, de modo geral, considerou os recursos adequados. Os genitores da família F4 percebem a maioria dos recursos como 'geralmente adequados'. A mãe, porém, considerou os recursos referentes ao tempo como 'nunca adequados' ou 'raramente adequados', enquanto o pai considerou 'nunca adequado' para os recursos financeiros, isto é, dinheiro para ser guardado.

\section{Discussão}

Ressalta-se, primeiramente, que os resultados descritos são limitados, devido à pequena quantidade de participantes e, aliado a este aspecto, já eram esperadas algumas diferenças entre as famílias, uma vez que estas são de contextos culturais distintos. É importante salientar que os recursos disponíveis às famílias residentes em Juiz de Fora são mais adequados do que aqueles disponíveis às famílias de Caxambu, sendo que um dos motivos para tal fato se refere ao tamanho de ambas as cidades. Esta 
última conta com 20,000 habitantes, enquanto Juiz de Fora tem aproximadamente 500,000 moradores. É evidente que as famílias experienciam contextos culturais e sociais distintos, bem como os recursos propiciados pelas instituições e profissionais são mais precários para as famílias da cidade pequena. Por exemplo, as adolescentes nas famílias F3 e F4, que moram na cidade pequena, nunca frequentaram o ensino fundamental e participam somente das atividades do centro especializado. Por outro lado, as adolescentes A1 e A2 frequentaram o ensino fundamental, participam de sessões de Fonoaudiologia, Psicologia, Psicopedagogia e Terapia Ocupacional. É evidente que os atendimentos são extremamente fundamentais para propiciar melhoria no desenvolvimento dessas participantes.

Além disso, os resultados sobre os eventos sociais a que as famílias participam mostram que as famílias F1 e F2, residentes em Juiz de Fora, frequentam mais eventos sociais do que as demais, realçando que F4 nunca frequenta cinema/teatro, festas típicas e centros culturais. É importante destacar também que F3 e F4 são as famílias com menor renda, o que dificulta o acesso aos recursos. Há que se considerar, por outro lado, o fato de estas famílias viverem em contextos culturais diversos, o que influencia nas crenças, valores e comportamentos dos genitores diante da filha com SD. Esse tema deve ser investigado por estudos futuros, uma vez que o modo como os genitores concebem a SD tem implicações no desenvolvimento do filho (Bastos \& Deslandes, 2009).

Ainda a respeito das atividades sociais frequentadas pelas famílias observa-se que as visitas familiares, os eventos em locais públicos/alimentação, as missas/cultos em geral e as festas típicas foram as mais relatadas. A literatura (Cunningham, 2008) aponta que muitos pais de pessoas com deficiência afirmam que se sentem isolados tanto social quanto emocionalmente. Cunningham (2008) afirma que o isolamento social implica em não conseguir sair e realizar diferentes atividades por causa do filho, enquanto o isolamento emocional está relacionado à sensação dos pais de não ter ninguém com quem compartilhar suas preocupações. Assim, Cunningham afirma que a principal fonte de apoio seria proveniente do cônjuge e de parentes próximos e os resultados do presente estudo corroboram essa afirmação.

No que se refere à dinâmica e funcionamento das famílias, Dessen e Braz (2005) afirmam que no final da década de 1960 o papel feminino mudou expressivamente inclusive na família, pois ocorreu um aumento na participação ativa das mulheres no contexto social, político e econômico, bem como nas relações sociais, passando estas a serem mais igualitárias entre os cônjuges e entre pais e filhos. Porém, isso acarretou em aumento do trabalho da mulher, pois além de exercer atividades profissionais elas ainda eram responsáveis pelos filhos (Biasoli-Alves, 2000). Wagner, Predebon, Mosmann e Verza (2005) destacam que, apesar de haver um crescimento no número de pais que compartilham com a mulher ou assumem a tarefa de educar os filhos, os cuidados e a educação dos filhos ainda são tarefas que não costumam ser compartilhadas pelo casal e os resultados do presente estudo corroboram com esse dado, incluindo também a família com maior renda, a qual é a única que dispõe do serviço de uma empregada.

Os resultados sobre as habilidades em atividades de vida diária (AVD) das adolescentes foram descritos de acordo com os relatos das mães e mostram que A2 e A4 são mais dependentes na realização de AVD's e A1 e A3 realizam mais atividades de maneira independente. Baseados no modelo bioecológico (Bronfenbrenner \& Ceci, 1994; Bronfenbrenner \& Morris, 1998), pode-se afirmar que tal fato relaciona-se às características da pessoa e do contexto, pois cada uma das adolescentes, apesar de ter a SD, possui características próprias e está inserida em contextos diferentes (cidade e família), os quais influenciam seu desenvolvimento. A interação entre a genética (síndrome de Down, temperamento, etc.), desenvolvimento cognitivo, emocional e social de cada participan- 
te, interação com microssistemas (escola, centro especializado etc.), além das características da dinâmica e funcionamento de cada família pode contribuir para os resultados no desenvolvimento e, portanto, para o desempenho das AVD's destas adolescentes. Assim, por exemplo, A1 frequentou a escola, centro especializado, vizinhança, entre outros microssistemas. Já A4, como foi verificado, frequenta poucos ambientes sociais e sua família possui escasso contato com amigos e parentes. Logo, seu desenvolvimento pode ter sido afetado tanto por suas características pessoais quanto pelo seu contexto familiar.

Glat e Freitas (2002) alertam para o fato de que algumas pessoas com DI possuem pequenas oportunidades de interagir livremente com pessoas do sexo oposto, o que limita a possibilidade de se relacionarem afetivamente. Glat e Freitas (2002) salientam ainda a crença existente na sociedade de que pessoas com DI são também deficientes emocionais. Luiz e Kubo (2007) concordam com a ideia de que pessoas com DI possuem possibilidades restritas de manterem relacionamentos amorosos, afirmando que, devido a tal restrição, essas pessoas tendem a fantasiar sobre ter um relacionamento amoroso. Seria interessante ter explorado a percepção dos genitores sobre os relacionamentos afetivos das filhas, já que as mães afirmaram que as adolescentes não possuíam namorados, mas não foram questionadas sobre o que pensavam a respeito da afetividade da filha, sobre as expectativas quanto aos relacionamentos afetivos das mesmas e sobre o desenvolvimento da sexualidade, o que pode ser explorado em estudos futuros.

Em relação ao apoio social, todas as famílias relataram que possuem apoio de instituições e de profissionais. Porém, a rede social familiar é bastante restrita, sendo, de um modo geral, composta predominantemente por cônjuge e filhos. Tal resultado é semelhante aos encontrados por Barbosa et al. (2009), apontando a família e os amigos como importantes fontes de apoio, bem como com os resultados obtidos por Nunes (2006), que apontam um maior índice de colaboração por parte do companheiro ou da companheira. Ainda em relação ao apoio social, os resultados obtidos sugerem que quanto maiores são os recursos disponíveis relacionados a abrigo, necessidade física, recursos pessoais, saúde, suporte intrafamiliar, cuidados com os filhos, comunicação e crescimento, ou seja, os aspectos avaliados pela Escala de Recursos da Família, menores são os suportes relacionados aos indivíduos e aos grupos, isto é, os recursos avaliados pela Escala de Apoio da Família. Assim, verifica-se que a mãe na família F1 apresentou menor pontuação na Escala de Recursos da Família e maior pontuação na Escala de Apoio da Família. Contraditoriamente, a mãe e o pai na família F2 apresentaram maior escore na Escala de Recursos da Família e menor escore na Escala de Apoio da Família e o pai na família F4 apresentou menor pontuação na Escala de Recursos da Família e pontuação mais alta na Escala de Apoio da Família. Esses resultados indicam que as famílias não têm apoios e recursos em níveis satisfatórios ao mesmo tempo, o que aponta para a necessidade da implementação de programas dirigidos a essas famílias nessa fase do ciclo de vida.

Quanto aos relatos dos genitores no tocante às reações e sentimentos vivenciados no momento do diagnóstico da filha, verifica-se que a reação mais manifestada diante da confirmação do diagnóstico foi 'aceitação', enquanto o sentimento mais relatado foi 'tristeza'. Já os sentimentos atuais em relação à filha com SD foram de 'insegurança', 'carinho' e 'felicidade'. No estudo de Pereira-Silva (2003) os genitores de crianças pré-escolares também manifestaram 'aceitação' diante da confirmação do diagnóstico e os sentimentos foram de 'revolta', 'sofrimento' e 'choque'. Diante da confirmação do diagnóstico todas as famílias relataram ter procurado ajuda, sendo que em três delas a mãe tomou a iniciativa e em apenas uma (F2) pai e mãe procuraram ajuda profissional juntos. Pereira-Silva e Dessen (2002) ressaltam que o momento da confirmação do diagnóstico merece uma atenção 
especial dos profissionais envolvidos, pois neste momento, os sentimentos se misturam e confundem os genitores, podendo gerar um desequilíbrio no funcionamento da família, com consequências futuras para o próprio desenvolvimento da criança com SD.

A maioria dos genitores relatou ter um 'conhecimento popular' sobre a SD, assemelhando-se aos resultados obtidos em outros estudos (Almeida, 2014; Pereira-Silva, 2003). No estudo de Almeida, oito genitores de crianças e adolescentes com SD (mães: $n=2,20 \%$; pais: $n=6,60 \%$ ) e dois irmãos (22\%) possuíam 'Conhecimento genérico' sobre a SD, enquanto nove genitores (mães: $n=6,60 \%$; pais: $n=3$ ) e um irmão tinham 'Conhecimento científico’. Segundo Almeida (2014) houve também aqueles que relataram 'Desconhecimento' sobre a síndrome (mães: $n=1,10 \%$; pais: $n=1$, $10 \%$; irmãos: $n=6,67 \%$ ). Diante disso, Pereira-Silva (2003) destaca a importância dos serviços oferecidos às famílias e seus filhos com DI ou com $\mathrm{SD}$, os quais devem considerar as características da população-alvo, bem como as peculiaridades de cada etiologia.

Destaca-se a necessidade de ocorrer mudanças nas concepções e planejamentos de pesquisas de forma a incluir toda a família e não somente o/a adolescente, como foco principal de análise, inserido em um contexto social específico, em determinado momento histórico. Vale ressaltar que é um desafio para o pesquisador investigar o contexto familiar, especialmente no Brasil, cujas famílias não têm tradição em pesquisa. Entretanto, fica o desafio para futuros pesquisadores que possam interessar-se por este tipo de investigação utilizando abordagem multimetodológica, buscando compreender a complexidade do desenvolvimento humano na área de família com filhos especiais.

\section{Referências}

Almeida, B. R. (2014). Interações fraternais em famílias com crianças e adolescentes com síndrome de Down. Dissertação de mestrado, Universidade Federal de Juiz de Fora, Juiz de Fora.

Amendola, F., Oliveira, M. A. C. \& Alvarenga, M. R. M. (2011). Influência do apoio social na qualidade de vida do cuidador familiar de pessoas com dependência. Revista da Escola de Enfermagem da USP, 45, 884-889.

Angélico, A. P. (2004). Estudo descritivo do repertório de habilidades sociais de adolescentes com sindrome de Down. Dissertação de mestrado, Universidade Federal de São Carlos, São Carlos. Araújo, Y. B., Reichert, A. P. S., Oliveira, B. R. G. \& Collet, N. (2011). Rede e apoio social de famílias de crianças com doença crônica: revisão integrativa. Ciência, Cuidado e Saúde, 10, 853-860.

Bailey, D. B. (2007). Introduction: Family adaptation to intellectual and developmental disabilities. Mental Retardation and Developmental Disabilities Research Reviews, 13, 291-292.

Barbosa, M. A. M., Pettengill, M. A. M., Farias, T. L. \& Lemes, L.C. (2009). Cuidado da criança com deficiência: suporte social acessado pelas mães. Revista Gaúcha de Enfermagem, 30, 406-412. Bardin, L. (2011). Análise de conteúdo. São Paulo: Edições 70.

Bastos, O. M. \& Deslandes, S. F. (2008). A experiência de ter um filho com deficiência mental: narrativas de mães. Cadernos de Saúde Pública, 24, 2141-2150.

Bastos, O. M. \& Deslandes, S. F. (2009). Adolescer com deficiência mental: a ótica dos pais. Ciência e Saúde Coletiva, 14, 79-87.

Biasoli-Alves, Z. M. M. (2000). Continuidades e rupturas no papel da mulher brasileira no século XX. Psicologia: Teoria e Pesquisa, 16, 233-239.

Bonomo, L. M. M., Garcia, A. \& Rossetti, C. B. (2009). O adolescente com síndrome de Down e sua rede de relacionamentos: um estudo exploratório sobre suas amizades. Psicologia: Teoria e Prática, 11, 114-130.

Bononi, B. M., Oliveira, A. C. V., Renattini, T. S. M., Sant'Anna, M. J. C., \& Coates, V. (2009). 
Síndrome de Down na adolescência: limites e possibilidades. Revista Adolescência e Saúde, 6, 51-56.

Bronfenbrenner, U. (2011). Bioecologia do desenvolvimento humano: tornando os seres humanos mais humanos. (A. Carvalho-BarretoTrad.). Porto Alegre: Artmed. (Trabalho originalmente publicado em 2005).

Bronfenbrenner, U. \& Ceci, S. J. (1994). Nature-nurture reconceptualized in developmental perspective: A bioecological model. Psychological Review, 101, 568-586.

Bronfenbrenner, U. \& Morris, P.A. (1998). The ecology of developmental processes. Em W. Damon \& R.M. Lerner (Orgs.), Handbook of child psychology: Vol. 1: Theoretical models of human development (pp. 993-1028). New York: Wiley.

Casarin, S. (2003). Aspectos psicológicos na Síndrome de Down. Em S. J. Schwartzman (Org.), Síndrome de Down (pp. 263-285). São Paulo: Memnon/Mackenzie.

Colnago, N. A. S. (2000). Orientação para pais de crianças com síndrome de Down: elaborando e testando um programa de intervenção. Tese de Doutorado, Programa de Pós-graduação em Psicologia, Universidade de São Paulo, Ribeirão Preto.

Cramm, J. M. \& Nieboer, A. P. (2011). Conditions affecting psychological distress among caregivers of children with intellectual disabilities seeking outside care and support using parental stress as a mediating factor. Journal of Intellectual Disabilities, 15, 101-113.

Cunningham, C. (1996). Families of children with Down Syndrome. Down Syndrome Research and Practice, 4, 87-95.

Cunningham, C. (2008). Sindrome de Down: uma introdução para pais e educadores. Porto Alegre: Artmed.

Dabrowska, A. \& Pisula, E. (2010) Parenting stress and coping styles in mothers and fathers of preschool children with autism and Down syndro- me. Journal of Intellectual Disability Research, 54, 266-280.

Dessen, M. A. (2009). Questionário de categorização do sistema familiar. Em L. Weber \& M. A. Dessen (Orgs.), Pesquisando a família: instrumentos para coleta e análise de dados (pp. 119-131). Curitiba: Juruá.

Dessen, M. A. \& Braz, M. P. (2000). Rede social de apoio durante transições familiares decorrentes do nascimento de filhos. Psicologia: Teoria e Pesquisa, 16, 221-231.

Dessen, M. A. \& Braz, M. P. (2005). A família e suas inter-relações com o desenvolvimento humano. Em M. A. Dessen \& A. L. Costa Jr. (Orgs.), $A$ ciência do desenvolvimento humano: tendências atuais e perspectivas futuras (pp. 113-131). Porto Alegre: Artmed.

Dezoti, A. P., Alexandre, A. M. C., Tallman, V. A. B., Maftum, M. A. \& Mazza, V. A. (2013). Rede social de apoio ao desenvolvimento infantil segundo a equipe de saúde da família. Escola Anna Nery Revista de Enfermagem, 17, 721-729.

Diniz, E., Garcia-Dias, A. C., Neiva-Silva, L., Nieto, C. J. \& Koller, S. H. (2012). Características familiares y apoyo percibido entre adolescentes brasileños con y sin experiencia de embarazo. Avances en Psicologia, 30, 65-80.

Douma, J. C. H., Dekker, M. C. \& Koot, H. M. (2006). Supporting parents of youths with intellectual disabilities and psychopathology. Journal of Intellectual Disability Research, 50, 570-581.

Dunst, C. J., Jenkins, V. \& Trivette, C. M. (1994). Measuring social support in families with young children with disabilities. Em C. J. Dunst, C. M. Trivette \& A. Deal (Eds.), Supporting and strengthening families: Methods, strategies and practices (pp. 152-160). Cambridge, UK: Brookline Books.

Eisenhower, A. \& Blacher, J. (2006). Multiple roles, ethnicity, and well-being among mothers of young adults with intellectual disability. Journal of Intellectual Disability Research, 50, 905-916. 
Falkenbach, A. P., Drexsler, G. \& Werler, V. (2008). A relação mãe/criança com deficiência: Sentimentos e experiências. Ciência \& Saúde Coletiva, 13, 2065-2073.

Flynt, S.W., Wood, T. A. \& Scott, R. L. (1992). Social support of mothers of children with mental retardation. Mental Retardation, 30, 233-236.

Glat, R. \& Freitas, R.C. (2002). Sexualidade e deficiência mental: pesquisando, refletindo e debatendo sobre o tema. Em R. Glat(Org.), Questões atuais em educação especial. Rio de Janeiro: Viveiros de Castro.

Goitein, P. C. \& Cia, F. (2011). Interações familiares de crianças com necessidades educacionais especiais: Revisão da literatura nacional. Revista Semestral da Associação Brasileira de Psicologia Escolar e Educacional, 15, 43-51.

Hall, S., Bobrow, M. \& Marteau, T. M. (1997). Parents attributions of blame for the birth of a child with Down Syndrome: A pilot study. Psychology and Health, 12, 579-587.

Hastings, R. P., Daley, D. \& Burns, C. (2006). Maternal distress and expressed emotion: cross-sectional and longitudinal relationships with behavior problems of children with intellectual disabilities. American Journal on Mental Retardation, 11, 48-61.

Head, L. \& Abbeduto, L. (2007). Recognizing the role of parents in developmental outcomes: A systems approach to evaluating the child with developmental disabilities. Mental Retardation and Developmental Disabilities Research Reviews, 13, 293-301.

Henn, C. G. \& Piccinini, C. A. (2010). A experiência da paternidade e o envolvimento paterno no contexto da síndrome de Down. Psicologia: Teoria e Pesquisa, 26, 623-631.

Henn, C. G., Piccinini, C. A. \& Garcias, G. L. (2008). A família no contexto de síndrome de Down: revisando a literatura. Psicologia em Estudo, 13, 485-493.

Hodapp, R. M., Burack, J. A. \& Zigler, E. (1998). Developmental approaches to mental retarda- tion: A short introduction. Em J. A. Burack, R. M. Hodapp \& E. Zigler (Eds.), Handbook of mental retardation and development. New York: Cambridge University Press.

Jussani, N. C., Serafim, D. \& Marcon, S. S. (2007). Rede social durante expansão da família. Revista Brasileira de Enfermagem, 60, 184-189.

Kreppner, K. (2000). The child and the family: Interdependence in developmental pathways. Psicologia: Teoria e Pesquisa, 16, 11-22.

Leet, H. E. \& Dunst, C. J. (1994). Measuring the adequacy of resources in households with young children. Em Carl J. Dunst, Carol M. Trivette \& Angela Deal (Eds.), Supporting and strengthening families: Methods, strategies and practices (pp. 105-114). Cambridge: Brookline Books.

Luiz, E. C. \& Kubo, O. M. (2007). Percepções de jovens com síndrome de Down sobre relacionar-se amorosamente. Revista Brasileira de Educação Especial, 13, 219-238.

Matsukura, T. S., Marturano, E. M., Oishi, J. \& Borasche, G. (2007). Estresse e suporte social em mães de crianças com necessidades especiais. Revista Brasileira de Educação Especial, 13, 415-428.

Moraes, J. R. M. M. \& Cabral, I. E. (2012). A rede social de crianças com necessidades especiais de saúde na (in)visibilidade do cuidado de enfermagem. Revista Latino-Americana de Enfermagem, 20, 282-288.

Most, D. E., Fidler, D. J., Laforce-Booth, C.,\& Kelly, J. (2006). Stress trajectories in mothers of young children with Down Syndrome. Journal of Intellectual Disability Research, 50, 501-514.

Nunes, C.C. (2006). Interação entre irmãos de indivíduos com deficiência mental: o papel da idade e do apoio social da família. Dissertação de Mestrado. Programa de Pós-graduação em Psicologia, Universidade Federal de São Carlos, São Carlos.

Oliveira, L. D. (2013) Famílias de adolescentes com deficiência intelectual: estresse, estratégias de enfrentamento e apoio social. Dissertação de 
Mestrado não-publicada, Programa de Pós-graduação em Psicologia, Universidade Federal de Juiz de Fora, Juiz de Fora, Brasil.

Oliveira, M. R. \& Dessen, M. A. (2012). Alterações na rede social de apoio durante a gestação e o nascimento de filhos. Estudos de Psicologia (Campinas), 29, 81-88.

Padeliadu, S. (1998). Time demands and experienced stress in Greek mothers of children with Down's syndrome. Journal of Intellectual Disability Research, 43, 144-153.

Pereira-Silva, N. L. (2003). Famílias de crianças com e sem sindrome de Down: um estudo comparativo das relações familiares. Tese de Doutorado não-publicada, Programa de Pós-graduação em Psicologia, Universidade de Brasília, Brasília, DF.

Pereira-Silva, N. L. \& Dessen, M. A. (2002). Síndrome de Down: etiologia, caracterização e impacto na família. Interação em Psicologia, 6, 167-176.

Pereira-Silva, N. L. \& Oliveira, L. D. (2011, novembro). Família e deficiência intelectual: pesquisas sobre estresse, estratégias de enfrentamento e apoio social. Trabalho apresentado no VIII Congresso Brasileiro de Psicologia do Desenvolvimento. Brasília: Associação Brasileira de Psicologia do Desenvolvimento Humano.

Sá, S. M. P. \& Rabinovich, E. P. (2006). Compreendendo a família da criança com deficiência física. Revista Brasileira de Crescimento e Desenvolvimento Humano, 16, 68-84.
Scabini, E. \& Ranieri, S. (2011). Família com filhos adolescentes: a perspectiva relacional. Em L. V. C. Moreira \& E. P. Rabinovich (Org.), Família e parentalidade: olhares da psicologia e da história (pp. 169-186). Curitiba: Juruá.

Siqueira, A. C., Betts, M. K. \& Dell'Aglio, D. D. (2006). Rede de apoio social e afetivo de adolescentes institucionalizados. Interamerican Journal of Psychology, 40, 149-158.

Soresi, S. \& Nota, L. (2000). A social skill training for persons with Down's syndrome. European Psychologist, 5, 33-43.

Stores, R., Stores, G., Fellows, B. \& Buckley, S. (1998). Daytime behaviour problems and maternal stress in children with Down's Syndrome, their siblings, and non-intellectually disabled and other intellectually disabled peers. Journal of Intellectual Disability Research, 42, 228-237.

Teixeira, F. C., \& Kubo, O. M., 2008. Características das interações entre alunos com síndrome de Down e seus colegas de turma no sistema regular de ensino. Revista Brasileira de Educação Especial, 14, 75-92.

Wagner, A., Predebon, J., Mosmann, C. \& Verza, F. (2005). Compartilhar tarefas? Papéis e funções de pai e mãe na família contemporânea. Psicologia: Teoria e Pesquisa, 21, 181-186.

Wodehouse, G. \& McGill, P. (2009). Support for family carers of children and young people with developmental disabilities and challenging behaviour: What stops it being helpful? Journal of Intellectual Disability Research, 53, 644-653.
Recebido: 16 de maio de 2014 Aprovado: 7 de outubro de 2014 
